\title{
AVALIAÇÃO DA ESPIROMETRIA NO PRÉ E PÓS OPERATÓRIO DE PACIENTES SUBMETIDAS A MASTECTOMIA TOTAL E QUADRANTECTOMIA NO HOSPITAL OPHIR LOYOLA
}

\author{
Ainá Patrícia Escorcio BARBOSA ${ }^{1}$ \\ Aline Vanessa Silva ANDRADE ${ }^{2}$ \\ Izabella Frias LOUREIRO
}

Antônio Rafael Wong RAMOS ${ }^{4}$

\begin{abstract}
${ }^{1}$ Graduada no curso de Bacharelado em Fisioterapia do Centro Universitário do Pará. ainapatricia @ hotmail.com ${ }^{2}$ Graduada no curso de Bacharelado em Fisioterapia do Centro Universitário do Pará. alineandrade.fisio@ hotmail.com ${ }^{3}$ Graduada no curso de Bacharelado em Fisioterapia do Centro Universitário do Pará. izabella_loureiro@ hotmail.com ${ }^{4}$ Fisioterapeuta, Mestre em Saúde, Sociedade e Endemias na Amazônia pelo Programa de Pós-Graduação Multidisciplinar na Universidade Federal do Amazonas, Universidade Federal do Pará e Fundação Oswaldo Cruz, Especialista em Fisioterapia Cardiorrespiratória e Docente do Curso de Fisioterapia do Centro Universitário do Pará. toninhoramos@icloud.com
\end{abstract}

Recebido em: 12/12/2016- Aprovado em: 15/08/2017 - Disponibilizado em: 30/12/2017

\begin{abstract}
RESUMO
Introdução: $\mathrm{O}$ índice de mulheres acometidas pelo câncer de mama tem aumentado tornando-se o segundo tipo mais frequente no mundo. $\mathrm{O}$ tratamento para este tipo de câncer difere entre diversos métodos, sendo o mais utilizado o procedimento cirúrgico destacando a quadrandectomia e a mastectomia. Pacientes submetidas ao procedimento cirúrgico tendem a apresentar alterações física sno período pós-operatório, podendo desenvolver complicações, sendo uma das mais frequentes a respiratória. Assim, é importante avaliar a função pulmonar, podendo ser realizada através do teste espirometrico. Objetivo: Avaliar a espirometria em pacientes submetidas a quadrantectomia e a mastectomia total unilateral no Hospital Ophir Loyola. Metodologia:Trata-se de uma pesquisa quantitativa de natureza observacional, prospectiva e descritiva, tendo amostra de 10 mulheres que realizaram quadrantectomia e 14 que realizaram mastectomia. Resultados: Verificou-se no pós operatório, média superior na cirurgia de mastectomia quando comparada a quadrantectomia para as variáveis CVF, VEF1, VEF1/CVF, enquanto a PFE apresentou média inferior. Houve discreta diminuição na maioria das variáveis diferindo apenas na CVF onde houve aumento do valor nas pacientes de mastectomia, enquanto que para as pacientes de quadrantectomia todas as variáveis apresentaram medias inferiores. Conclusão: $O$ estudo concluiu que não houve alterações pulmonares significativas quando comparado as cirurgias de mastectomia e quadrantectomia. O mesmo foi observado referente as cirurgias de mastectomia e quadrantectomia quando comparados o pré e o pós-operatório, individualmente. Em virtude disso, sugerem-se novas pesquisas voltadas a investigação do comprometimento da função pulmonar advinda da cirurgia de mastectomia e quadrantectomia.
\end{abstract}

Palavras-chaves: Neoplasias da mama. Espirometria. Fisioterapia. Mastectomia. Mastectomia Segmentar.

\begin{abstract}
Introduction: The rate of breast cancer has increased, becoming the second most common type worldwide. The treatment for this type of cancer differs between several methods, being surgery the most used, emphasizing quadrandectomy and mastectomy. Patients submitted to the surgical procedure tend to present physical alterations in the postoperative period, being able to develop complications, one the most frequent being the respiratory one. Thus, it is important to evaluate pulmonary function which can be done through the spirometric test. Objective: To evaluate spirometry in patients who underwent quadrantectomy and unilateral total mastectomy at Hospital Ophir Loyola. Methodology: This is a quantitative research of an observational, prospective and descriptive nature, with a sample of 10 women who underwent a quadrantectomy and 14 who underwent a mastectomy. Results: The mean postoperative time in the mastectomy group was higher regardind FVC, FEV1, FEV1/FVC, than in the quadrantectomy group, while the PEF presented a lower mean. There was a slight decrease in the majority of the variables differing only in FVC where there was an increase in the value in the mastectomy patients, while in the quadrantectomy patients all the variables had lower mean values. Conclusion: The study concluded that there were no significant pulmonary alterations when compared mastectomy and quadrantectomy surgeries. The same was observed regarding the surgeries of
\end{abstract}


mastectomy and quadrantectomy when compared the pre and postoperative, individually. Therefore, new research is suggested to investigate the involvement of lung function in mastectomy and quadrantectomy surgery.

Keywords: Breast Neoplasms. Spirometry. Physical Therapy Specialty. Mastectomy. Segmental.

\section{INTRODUÇÃO}

O câncer é caracterizado como uma enfermidade crônica que se origina da proliferação descontrolada de células.

Quando retratado o câncer de mama, o mesmo pode estar relacionado à interação de diferentes fatores, dentre eles, os fatores genéticos, o estilo de vida, os hábitos reprodutíveis e o meio ambiente, sendo uma doença que possui um alto poder de propagação induzindo ao pensamento de debilidades e mutilações, gerando assim certo impacto nos âmbitos físico, psicológico e estético do indivíduo (BREGAGNOL; DIAS, 2010; INUMARU; SILVEIRA; NAVES, 2011; LOUREIRO et al 2012).

O Brasil está entre os países com maior acometimento deste tipo de câncer, sendo responsável por $22 \%$ dos novos casos a cada ano e ainda se torna o maior causador de morte da população feminina brasileira (BREGAGNOL; DIAS, 2010; URBAN et al 2012; ABREU et al 2014; ROCHA et al 2014).

O tratamento para o câncer de mama difere entre diversos métodos, realizado através da radioterapia, quimioterapia, hormonioterapia e cirurgias, que dependerá do estadiamento clinico e do tipo histológico do tumor, é o tratamento mais utilizado o procedimento cirúrgico, destacando a quadrandectomia que é a retirada de um quadrante ou parte da mama, onde está localizado o tumor, desde que este apresente dimensões mínimas entre 2 a $2,5 \mathrm{~cm}$ e a mastectomia que caracteriza-se pela retirada total da mama (ABREU et al 2014; ROCHA et al 2014).

Pacientes submetidas ao procedimento cirúrgico tendem a apresentar alterações físicas no período pós-operatório, podendo desenvolver complicações, sendo uma das mais frequentes as respiratórias, que podem ocorrer em até $22 \%$ dos casos, devido à proximidade entre o local em que é realizado a cirurgia e a região pulmonar, porconta de fibroses ou aderências cicatriciais bem como, as retrações musculares, hipoventilação decorrente da dor e do medo, imobilização,depressão do sistema nervoso central, disfunção diafragmática temporária e fraqueza da musculatura ventilatória que interferem nas trocas gasosas e reduzem as funções respiratórias(BREGAGNOL; DIAS, 2009; LOUREIRO et al 2012; ABREU et al 2014).

Dentre as consequências respiratórias associadas as cirurgias para tratamento do câncer de mama estão a diminuição da capacidade vital (CV) em cerca de 50 a 60\%, da capacidade residual funcional (CRF) cerca 
de $30 \%$, dos volumes de reserva inspiratório (VRI) e expiratório (VRE) e dos fluxos expiratórios,em função da redução da atividade diafragmática, sendo observadas nas primeiras 16 a 24 horas do pósoperatório(SAAD; ZAMBOM, 2001; DIAS et al 2008).

A função pulmonar pode ser avaliada por meio da espirometria que é classificada como um exame funcional respiratório para detectar e diagnosticar diversas disfunções pulmonares. E tem a capacidade de medir diferentes volumes pulmonares principalmente a capacidade vital forçada (CVF) e volume expiratório forçado no primeiro segundo (VEF1), realizado através de uma inspiração profunda seguido de uma expiração profunda onde esse volume expirado será mensurado (COSTA; JAMAMI, 2001; SILVA et al 2005; ATEMADINEZHAD; ALIZADEH, 2011).

Tendo em vista o contingente populacional de mulheres acometidas com câncer de mama, a realização de tratamento cirúrgico por conta do agravo da doença, juntamente com os achados na literatura, que relatam que a função pulmonar nessas pacientes é afetada em ambas as formas de tratamento, este estudo tem como objetivo avaliar a espirometria em pacientes submetidas a quadrantectomia e a mastectomia total unilateral no Hospital Ophir Loyola, observar os efeitos da mastectomia e da quadrantectomia sobre o pico de fluxo expiratório, volume expiratório forçado no primeiro segundo, capacidade vital forçada e o Índice de Tiffenou e comparar os valores obtidos.

\section{MATERIAIS E METODOS}

O estudo caracteriza-se como quantitativo de natureza observacional, prospectiva e descritiva no Hospital Ophir Loyola, no período de Junho a Outubro de 2016. Realizado após aprovação do comitê de ética do Centro Universitário do Pará CESUPA (CAAE: 52381115.2.0000.5169) e do Hospital Ophir Loyola - HOL (CAAE: 52381115.2.3001.5550).

A população deste estudo foi composta por 24 mulheres que foram submetidas ao tratamento cirúrgico sendo que 10 mulheres realizaram cirurgia de quadrantectomia e 14 realizaram cirurgia de mastectomia, com faixa etária de 31 a 81 anos, que não faziam tratamento quimioterapêutico ou radioterapêutico, e não houvessem realizado alguma cirurgia associada ou apresentassem alguma complicação ou disfunção respiratória, após a assinatura do Termo de Consentimento Livre e Esclarecido (TCLE).

Foi realizada a espirometria, utilizando o aparelho da marca Clemente One Flow, no período pré-operatório e no primeiro dia de pós operatório, sendo os dados coletados e organizados em fichas elaboradas pelas 
autoras, contendo as seguintes variáveis: idade, sexo, patologia de origem e os resultados obtidos pelo espirômetro sendo estes capacidade vital forçada (CVF), volume expiratório forçado no primeiro segundo (VEF1), pico de fluxo expiratório (PFE) e o Índice de Tiffeneau (VEF1/CVF).

As avaliações foram realizadas, pelas autoras, sendo realizada pela mesma pessoa no pré e pós-operatório, com a paciente na posição sentada, com o tronco em ângulo de $90^{\circ}$, braços relaxados na lateral do tronco. Foi solicitada a colocação de um clip nasal e o acoplamento dos lábios ao bocal do espirometro, sendo solicitada que realizasse uma inspiração forçada seguida de uma expiração forçada, repetindo o procedimento 3 vezes, adotando-se o maior valor.

Foram tabulados os dados de acordo com a natureza das variáveis, onde se realizou análise descritiva. O banco de dados, bem como as tabelas e os gráficos foram construídos no Microsoft EXCEL.

A análise estatística foi realizada utilizando o programa SPSS 20.0, com base em medidas de tendência e dispersão para as variáveis.As comparações das variáveis foram feitas através do teste $t-$ Student e para a análisedo pico de fluxo expiratório (PFE), volume expiratório forçado no primeiro segundo (VEF1), capacidade vital forçada (CVF) e o Índice de Tiffenou (VEF1/CVF), segundo o tipo de cirurgia, todos ao nível de significância estatística de 0,05. O teste da
ANOVA (Análise de Variância) foi utilizado para determinar se os valores pulmonares encontrados nas pacientes de mastectomia e de quadrantectomia diferiram entre si.

\section{RESULTADOS}

O teste de Análise de Variância (ANOVA - One Way)mostrou que, as médias para pico de fluxo expiratório (PFE), volume expiratório forçado no primeiro segundo (VEF1), capacidade vital forçada (CVF) e o Índice de Tiffenou (VEF1/CVF) no pósoperatório, não apresentaram diferença significativa $(\mathrm{p}>0.05)$ entre os grupos.

No caso do PFE no pós-operatório, o grupo mastectomia obteve média superior $(\boldsymbol{\mu}$ $=85.7)$ ao grupo quadrantectomia $(\mu=$ 65.90), porém não é uma diferença significativa ( $p>0.05)$ (tabela 1$)$. Na variável CVF no pós-operatório, o grupo mastectomia obteve média superior $(\boldsymbol{\mu}=\mathbf{2 . 1 4 6})$ ao grupo quadrantectomia $(\mu=\mathbf{1 . 6 7 3})$, porém não é uma diferença significativa ao nível de 0.05, mas ao nível de 0.10 é uma diferença significativa (tabela 1).No que se refere ao VEF1 no pós-operatório, o grupo mastectomia obteve média superior $(\boldsymbol{\mu}=$ 1.136) ao grupo quadrantectomia $(\boldsymbol{\mu}=\mathbf{0 . 9 1 6})$, porém não é uma diferença significativa ( $>>0.05$ ) (tabela 1). No caso do VEF1/CVF no pós operatório, o grupo mastectomia obteve média superior $(\boldsymbol{\mu}=\mathbf{5 8 . 7 1})$ ao grupo 
quadrantectomia $(\boldsymbol{\mu}=\mathbf{5 5 . 1 0})$, porém não é

$1)$.

uma diferença significativa $(\mathrm{p}>0.05)$ (tabela

Tabela 1 - Distribuição dos pacientes e teste de comparação de médias submetidos à Quadrantectomia e a Mastectomia, segundo a idade, pico de fluxo expiratório (PFE), volume expiratório forçado no primeiro segundo (VEF1), capacidade vital forçada (CVF) e o Índice de Tiffenou (VEF1/CVF).

\begin{tabular}{cccccccccc}
\hline \hline Variável & Grupo & Média & DP & Mínimo & Q1 & Mediana & Q3 & Máximo & P-valor \\
\hline PFE - Pré & Mastectomia & 103.9 & 53.9 & 20.0 & 57.5 & 105.0 & 138.8 & 220.0 & \\
Operatório & Quadrantectomia & 71.9 & 33.1 & 25.0 & 48.8 & 62.5 & 95.0 & 135.0 & 0.110 \\
\hline CVF - Pré & Mastectomia & 1.807 & 0.673 & 0.750 & 1.288 & 1.700 & 2.213 & 3.300 & \\
Operatório & Quadrantectomia & 1.915 & 0.881 & 0.550 & 1.125 & 1.975 & 2.425 & 3.600 & 0.737 \\
\hline VEF1 - Pré & Mastectomia & 1.271 & 0.577 & 0.200 & 0.813 & 1.350 & 1.712 & 2.150 & \\
Operatório & Quadrantectomia & 0.975 & 0.495 & 0.200 & 0.587 & 0.925 & 1.325 & 1.900 & 0.203 \\
\hline VEF1/CVF - & Mastectomia & 71.64 & 25.56 & 16.00 & 47.75 & 81.00 & 93.00 & 97.00 & \\
Pré Operatório & Quadrantectomia & 57.30 & 30.21 & 17.00 & 32.25 & 51.00 & 89.50 & 99.00 & 0.222 \\
\hline PFE - Pós & Mastectomia & 85.7 & 43.2 & 35.0 & 45.0 & 80.0 & 113.8 & 190.0 & \\
Operatório & Quadrantectomia & 65.90 & 27.00 & 25.00 & 50.50 & 60.00 & 86.25 & 120.00 & 0.214 \\
\hline CVF - Pós & Mastectomia & 2.146 & 0.660 & 1.050 & 1.480 & 2.125 & 2.728 & 3.100 & \\
Operatório & Quadrantectomia & 1.673 & 0.566 & 0.980 & 1.250 & 1.600 & 1.938 & 2.900 & $0.080 *$ \\
\hline VEF1 - Pós & Mastectomia & 1.136 & 0.468 & 0.450 & 0.850 & 1.050 & 1.487 & 2.150 & \\
Operatório & Quadrantectomia & 0.916 & 0.389 & 0.300 & 0.675 & 0.850 & 1.162 & 1.700 & 0.238 \\
\hline VEF1/CVF - & Mastectomia & 58.71 & 25.51 & 17.00 & 34.00 & 56.50 & 85.00 & 95.00 & \\
Pós Operatório & Quadrantectomia & 55.10 & 20.61 & 21.00 & 30.75 & 63.50 & 68.75 & 77.00 & 0.715 \\
\hline \hline
\end{tabular}

(1) Teste da ANOVA ( $\mathrm{p}<0.05)$.

* As médias diferem significativamente ao nível de $0.10(\mathrm{p}<0.10)$

Fonte: Elaborado pelas autoras (2016)

\section{O teste $\mathrm{t}$ de Student para comparação} de médias mostra que, as médias para PFE, VEF1, CVF e o VEF1/CVF não apresentam diferença significativa ( $p>0.05)$ no pré e pósoperatório para pacientes submetidos à Mastectomia.

No caso do PFE, a média no pósoperatório é inferior $(\boldsymbol{\mu}=\mathbf{8 5 . 7})$ ao préoperatório $(\mu=103,93)$, porém não é uma diferença significativa $(p>0.05)$. A variável CVF apresentou média no pós-operatório superior $(\boldsymbol{\mu}=\mathbf{2 , 1 5})$ ao pré-operatório $(\boldsymbol{\mu}=$ 1,81), porém não é uma diferença significativa $(p>0.05)$. No que refere ao VEF1, a média no pós-operatório é inferior ( $\mu$ $=1,14)$ ao pré-operatório $(\boldsymbol{\mu}=\mathbf{1 , 2 7})$, porém não é uma diferença significativa ( $\mathrm{p}>0.05)$. No caso do VEF1/CVF a média no pósoperatório é inferior $(\boldsymbol{\mu}=\mathbf{5 8 , 7 1})$ ao préoperatório $(\mu=\mathbf{7 1 , 6 4})$, porém não é uma diferença significativa $(\mathrm{p}>0.05)$.

Utilizando, ainda, o teste t de Student para comparação de médias, esta mostra que, as médias para PFE, VEF1, CVF e o VEF1/CVF não apresentam diferença significativa $(p>0.05)$ no pré e pós-operatório dos pacientes submetidos a Quadrantectomia.

No que se refere ao PFE, a média no pós-operatório é inferior $(\boldsymbol{\mu}=\mathbf{6 5 , 9 0})$ ao préoperatório $(\boldsymbol{\mu}=\mathbf{7 1 , 9 0})$, porém não é uma diferença significativa $(p>0.05)$. A variável CVF apresentou média no pós-operatório 
inferior $(\mu=1,67)$ ao pré-operatório $(\mu=$ 1,92), porém não é uma diferença significativa $(p>0.05)$. Quanto ao VEF1 a média no pós-operatório é inferior $(\mu=$ $\mathbf{0 , 9 2})$ ao pré-operatório $(\boldsymbol{\mu}=\mathbf{0 , 9 8})$, porém não é uma diferença significativa $(\mathrm{p}>0.05)$. No caso do VEF1/CVF a média no pósoperatório é inferior $(\boldsymbol{\mu}=\mathbf{5 5 , 1 0})$ ao préoperatório $(\boldsymbol{\mu}=\mathbf{5 7 , 3 0})$, porém não é uma diferença significativa $(\mathrm{p}>0.05)$.

\section{DISCUSSÃO}

As cirurgias para o tratamento de câncer de mama tendem a ocasionar no pósoperatório complicações pulmonares por limitação da expansibilidade torácica, por conta da dor, medo, aderência cicatricial, fraqueza da musculatura ventilatória o que tende a ocasionar menor ventilação do pulmão e assim o comprometimento pulmonar, por esse motivo este estudo objetivou avaliar a espirometria em pacientes submetidas a quadrantectomia e a mastectomia total unilateral no Hospital Ophir Loyola.

Esta pesquisa, avaliou a função pulmonar em 14 pacientes submetidas a mastectomia total unilateral,com média de idade de 53.43 e, 10pacientes que realizaram a quadrantectomia, com média de idade de 55.40 , comparando os valores obtidos no préoperatório e no primeiro dia de pós-operatório referente a cada cirurgia, de modo individual, e comparando ambos os procedimentos.
No presente estudo foi observado que as variáveis CVF, VEF1, VEF1/CVF apresentaram média superior na cirurgia de mastectomia quando comparadas à cirurgia de quadrantectomia, enquanto que a variável PFE apresentou média inferior na cirurgia de mastectomia quando comparado à cirurgia de quadrantectomia, ambas levando em consideração o resultado no pós-operatório, porém em nenhum dos resultados houve diferença significativa.

Segundo Abreu et al (2014), uma pesquisa com 20 mulheres, sendo estás dividas em 3 grupos onde o grupo 1 foi formado por mulheres que realizaram a cirurgia de mastectomia radical, o grupo 2, mulheres que realizaram a cirurgia de mastectomia + reconstrução mamaria com prótese e o grupo 3, mulheres que realizaram a cirurgia de quadrantectomia, destacando-se que ao comparar os três grupos entre si, houve uma homogeneidade na análise, devido em todos os tipos de atos cirúrgicos para tratamento de câncer de mama ocorrer fraqueza da musculatura ventilatória e a disfunção diafragmática acarretando a diminuição das funções respiratórias e a semelhança entre os valores.

Silva, Gazzana e Knorst (2010), corroborando com o descrito por Abreu et al (2014), descrevem que pacientes submetidos a cirurgia torácica, em geral, tendem a apresentar 12 a $70 \%$ de complicações pulmonares sendo tais complicações advindas 
de disfunções dos músculos respiratórios e a outras mudanças na mecânica da parede torácica, o que leva à redução dos volumes ventilatórios, justificando a homogeneidade nos resultados entre mastectomia e quadrantectomia, tendo em vista que ambos os procedimentos cirúrgicos manifestaram as mesmas complicações respiratórias.

Nesta pesquisa, referente ao resultado na cirurgia de mastectomia, houve discreta diminuição das medias no pós-operatóriodas variáveis PFE, VEF1e na relação VEF1/CVF, diferindo os resultados da variável CVF em que houve um aumento da média no pósoperatório quando comparado ao préoperatório, ambos os resultados sem apresentar diferença significativa. Foi observado, no momento da reavaliação, após o procedimento cirúrgico,que as pacientes encontravam-se mais dispostas e motivadas a realizar o teste, quando comparado ao momento da avaliação pré-operatória, o que pode justificar o aumento, discreto, da variável CVF no pós-operatório pode tal fato ser ratificado pelo estudo de Contesini, Garcia Jr e Caromano (2011), que destaca que diversos fatores externos, como fatores emocionais e o comportamento, podem influenciar na dinâmica estabelecida entre o pulmão, a caixa torácica e a musculatura,o que levam a alterações dos parâmetros ventilatórios, e ainda reiterado pela pesquisa de Pereira (2002), ao descrever a respeito do teste esferométrico onde, também, destaca-se que a motivação do paciente ao realizar a avaliação pode influenciar no resultado do teste.

Ainda se tratando da cirurgia de mastectomia, segundo Santos et al (2013),ao realizarem um estudo avaliando a espirometria,em mulheres submetidas a cirurgia de mastectomia, sendo analisados a capacidade vital forçada, volume expiratório forçado, o pico de fluxo expiratório e relação VEF1/CVF,foi observado diminuição significativa da CVF (23,52\%), da VEF1 $(26,23 \%)$ e da PFE $(13,12 \%)$ enquanto que a relação VEF1/CVF não apresentou alteração significativa. No entanto, quatro, das participantes do estudo já haviam entrado em contato com agentes que alteram a função pulmonar, sendo um deles o cigarro, onde segundo NEGREIROS (2010), quanto mais prolongada for a ação tabagista maior será o comprometimento pulmonar, o que pode justificar a diminuição significativa dos valores.

Estudos semelhantes realizados por Bregagnol e Dias (2010), evidenciaram que ao avaliar 28 mulheres submetidas à cirurgia de mastectomia, houve diminuição dos valores das variáveis CVF e VEF1, porém no estudo em questão foi observado também a diminuição na força muscular, quando avaliado, ratificando o resultado de Loureiro et al (2012), justificando que a proximidade da cirurgia com o pulmão ocasiona um déficit muscular na biomecânica respiratória, 
afetando os volumes e capacidades

pulmonares.

Em relação à cirurgia de quadrantectomia, observou-se, neste estudo, que nas variáveis PFE, CVF, VEF1, VEF1/CVF as médias apresentadas no pósoperatório foram inferiores às apresentadas no pré-operatório, porém, ambas as médias não apresentaram diferença significativa.

No estudo realizado por Santos et al (2013), em que constatou ao avaliar, também, pacientes que foram submetidas a cirurgia de quadrantectomia, houve uma diminuição significativa nos valores das variáveis CVF, PFE, VEF1, não apresentando alteração significativa na relação VEF1/CVF. Esses dados foram ratificados pelo estudo de Sampaio (2015), ao afirmar que as complicações respiratórias causadas pelos procedimentos cirúrgicos alteram a função respiratória pela localização cirúrgica na parede torácica, presença de aderências, alterações da musculatura como peitoral maior e menor, subclávio e intercostais internos e externos, contribuindo para a diminuição significativa nos valores de tais variáveis.

Apesar dos estudos citados, existe uma escassez de pesquisas abordando a respeito das complicações pulmonares advindas da cirurgias de quadrantectomia, o que dificulta o aprofundamento e o entendimento, real, a respeito das complicações pulmonares que tal procedimento pode manifestar.

\section{CONCLUSÃO}

Diante do exposto, conclui-se que não houve alterações pulmonares significativas quando comparados os tipos cirúrgicos de mastectomia e quadrantectomia e nas pacientes que realizaram cirurgia de mastectomia e quadrantectomia quando comparado o pré e o pós-operatório de cada cirurgia.

A literatura encontrada para abordar o assunto, relata que quando comparado o pré e o pós-operatório de ambas as cirurgia, ocorre uma diminuição significativa nos valores das variáveis avaliadas. Em virtude disso, sugerem-se novas pesquisas voltadas para a investigação do comprometimento da função pulmonar advinda da cirurgia de mastectomia e quadrantectomia.

\section{REFERENCIAS BIBLIOGRAFICAS}

ABREU, A. P. M. et al. Função pulmonar e força muscular respiratória em pacientes submetidas à cirurgia oncológica de mama. Revista Brasileira de Cancerologia, vol. 60, n. 2, p. 151-157, 2014. Disponível em: < http://www1.inca.gov.br/rbc/n_60/v02/pdf/09 -artigo-funcao-pulmonar-e-forca-muscularrespiratoria-em-pacientes-submetidas-acirurgia-oncologica-de-mama.pdf $>$. Acesso em: 26/06/15.

BREGAGNOL, R. K; DIAS, A. S. Alterações funcionais em mulheres submetidas à cirurgia de mama com linfadenectomia axilar total.

Revista Brasileira de Cancerologia, vol. 56, n.1, p. 25-33, 2010. Disponível em: <http://www.inca.gov.br/rbc/n_56/v01/pdf/05 _artigo_alteracoes_funcionais_linfadenectomi a.pdf>. Acesso em: 26/05/15. 
CONTESINI, A. M; GARCIA JR, A;

CAROMANO, F. A. Influência das variações da postura sentada na função respiratória: revisão de literatura. Fisioter Mov, vol. 24, n. 4, p. 757 - 767, out./dez., 2011. Disponível em:

<http://www.scielo.br/pdf/fm/v24n4/21.pdf >. Acesso em: 21/11/16

COSTA, D; JAMAMI, M. Bases fundamentais da espirometria. Rev. bras. fisioter, vol. 5, n. 2, p. 95-102, 2001. Disponível em: <http://rbfbjpt.org.br/files/v5n2/v5n2a07.pdf >. Acesso em: 31/05/15.

DIAS, C. M. et al. Inspirometria de incentivo e breath stacking: repercussões sobre a capacidade inspiratória em indivíduos submetidos à cirurgia abdominal. Rev. bras. fisioter, São Carlos, vol. 12, n. 2, p. 94-99, mar./abr. 2008. Disponível em: <http://www.scielo.br/pdf/rbfis/v12n2/a04v12 n2.pdf>. Acesso em: 26/05/15.

\section{ETEMADINEZHAD, S; ALIZADEH, A,} 2011. Valores de referência para espirometria em adultos saudáveis na província de Mazandaran, Irã. J Bras Pneumol., São Paulo, vol. 37, n. 15, p. 615-625, set./out. 2011. Disponível em: <http://www.scielo.br/scielo.php?pid=S180637132011000500008\&script=sci_arttext $>$. Acesso em: 30/07/15.

INUMARU, L. E; SILVEIRA, E. A; NAVES, M. M. V. Fatores de risco e de proteção para câncer de mama: uma revisão sistemática.

Cad. Saúde Pública, Rio de Janeiro, vol. 27, n. 7, p. 1259-1270, jul. 2011. Disponível em: <http://www.scielo.br/pdf/csp/v27n7/02.pdf>. Acesso em: 31/05/15.

LOUREIRO, L. P. et al. Incidência de complicações pulmonares em mulheres mastectomizadas no pós operatório imediato.

Revista Ensaio e Ciência: Ciências Biológicas, Agrárias e da Saúde, vol. 16, n. 1, p. 95-107, 2012. Disponível em: <http://www.redalyc.org/articulo.oa?id=2602 5372007>. Acesso em: 31/05/15.
NEGREIROS, A. S. B. A influência do tabagismo na prova de função pulmonar e no estado nutricional em idosos. 2010. 88f. Dissertação (Mestrado em Geriatria). Faculdade de Medicina da Universidade de Coimbra, Portugal, 2010. Disponível em: $<$ https://estudogeral.sib.uc.pt/bitstream/10316 /14207/1/A\%20influ\%C3\%AAncia\%20do\%2 0tabagismo $\% 20$ na $\% 20$ prova $\% 20$ de $\% 20$ fun $\%$ C3\%A7\%C3\%A3o\%20pulmonar.pdf >. Acesso em: 12/11/16.

PEREIRA, C. A. C. Espirometria. J

Pneumol, vol. 28, supl. 3, out., 2002.

Disponível

em: <http://www.jornaldepneumologia.com.br /PDF/Suple_139_45_11\%20Espirometria.pdf >. Acesso em: 21/11/2016.

ROCHA, M. S. et al. Avaliação funcional pré e pós-cirurgia conservadora da mama e linfadenectomia axilar: relato de caso.

Disciplinarum Scientia, Santa Maria, vol. 15, n. 1, p. 55-64, 2014. Disponível em: <http://www.periodicos.unifra.br/index.php/di sciplinarumS/article/view/1065/1009>. Acesso em: 01/06/15.

SAAD, I. A. B; ZAMBOM, L. Variáveis clínicas de risco pré-operatório. Rev Ass Med Brasil, vol. 47, n. 2, p. 117-124, 2001.

Disponível em: <http://www.scielo.br/pdf/ramb/v47n2/a29v4 7n2.pdf>. Acesso em: 01/06/15.

SANTOS, D. E. et al. Efeito da Radioterapia na função pulmonar e na fadiga de mulheres em tratamento para o câncer de mama.

Fisioter Pesq, vol. 20, n. 1, p. 50-55, 2013. Disponível em:

<http://www.scielo.br/pdf/fp/v20n1/09.pdf >. Acesso em: 04/11/2016.

SAMPAIO, S. S. S. Parâmetro de avaliação cinético-funcional e ventilatório no tratamento de câncer de mama. 2015. 46f. Trabalho de conclusão de curso (Graduação em Fisioterapia). Universidade Estadual da Paraíba, 2015. Disponível em:

<http://dspace.bc.uepb.edu.br/jspui/bitstream/ 123456789/10838/1/PDF\%20- 
$\%$ 20Sabrinne $\% 20$ Suellen $\% 20$ Santos $\% 20$ Sam paio.pdf>. Acesso em: 26/10/2016

SILVA, L. C. C. et al. Epirometria na prática médica. Revista AMRIGS, Porto Alegre, vol, 49, n. 3, p. 183-194, jul./set. 2005. Disponível em: <http://www.amrigs.com.br/revista/4903/espirometria.pdf>. Acesso em: 02/06/15.

SILVA, D. R; GAZZANA, M. B; KNORST, M. M. Valor dos achados clínicos e da avaliação funcional pulmonar pré-operatórios como preditores das complicações pulmonares pós-operatórias. Rev Assoc Med Bras, vol. 56, n. 5, p. $551-557,2010$. Disponível em:

<http://www.scielo.br/pdf/ramb/v56n5/v56n5 a16>. Acesso em: 11/11/216.

URBAN, L. A. B. D. et al. Recomendações do colégio brasileiro de radiologia e diagnóstico por imagem, da sociedade brasileira de mastologia e da federação brasileira das associaçõesde ginecologia e obstetrícia para rastreamento do câncer de mama por métodos de imagem. Revista

Radiol Bras, vol. 45, n. 6, p. 334-339, nov./dez. 2012. Disponível em: <http://www.scielo.br/pdf/rb/v45n6/09.pdf>. Acesso em: 02/06/15. 